ITM Web of Conferences 1, 01002 (2013)

DOI: $10.1051 /$ itmconf/ 20130101002

(C) Owned by the authors, published by EDP Sciences, 2013

\title{
Weighted Activity and Costing of Surveillance and Control in Animal Epidemiology
}

\author{
Raphaël Duboz ${ }^{1,2, a}$ \\ ${ }^{1}$ CIRAD, Centre Internationnal de Recherche Agronomique pour le Développement, UPR Agirs, Campus \\ International de Baillarguet, 34398 Montpellier Cedex 5, France. \\ ${ }^{2}$ AlT, Asian Institute of Technology, Computer Science \& Information Management, P.O. Box 4, Klong Luang, \\ Pathumthani 12120, Thailand.
}

\begin{abstract}
Activity based modeling and simulation is a very promising field. It has recently demonstrated its potential from modeling software development methods [9] to the design of low energy sensors. In this position paper, I would like to initiate a reflexion on the use of the weighted activity in the context of financial costing using the formulation recently proposed by X. Hu and B.P. Zeigler [5]. I propose here to formalize a recent approach of costing, the Time-Driven Activity Based Costing [3], using the theoretical modeling framework initiated by B.P. Zeigler [6]. I argue that this specification can potentially improve the traditional model of Time-Driven Activity Based Costing. I illustrate the approach in the context of surveillance and control in animal epidemiology. The demonstration of its effectiveness is not done in this position paper. Nevertheless, as the existing costing systems are designed to support decision making in business management, the scenario based approach proposed in this paper should be very useful to support decisions in complex management situations.
\end{abstract}

\section{Introduction}

The epidemiological surveillance and the control of the spread of contagious diseases within an animal population is a complex process. Epidemiological surveillance can be defined as an observation method based on continuous recordings and analysis of epidemiological data. Epidemiological surveillance tracks the health status or the risk factors of a well defined population in order to detect the emergence of a disease, to keep a check on an epizootic ${ }^{1}$ situation, or to monitor the dynamic of an endemic ${ }^{2}$ disease. One objective of the epidemiological surveillance is to provide useful data to decision makers so they can choose to trigger, or not, control actions for the mitigation of the disease spread [1]. The control measures can be quarantine measures, restriction of trading, or culling for instance. The surveillance and control processes involve numerous actors, and are very complex. Actors are the farmers, the veterinarians, the breeding technicians, biological analysis laboratories, policy makers, etc. We can also distinguish the passive surveillance from the active one, which are the spontaneous declaration of cases by the farmers, vets or breeding technicians for instance, and

\footnotetext{
ae-mail: raphael.duboz@cirad.fr

1 "Epizootic" in an animal population stands for "epidemic" in the human population.

${ }^{2}$ Already present in a population for a certain period of time.
} 


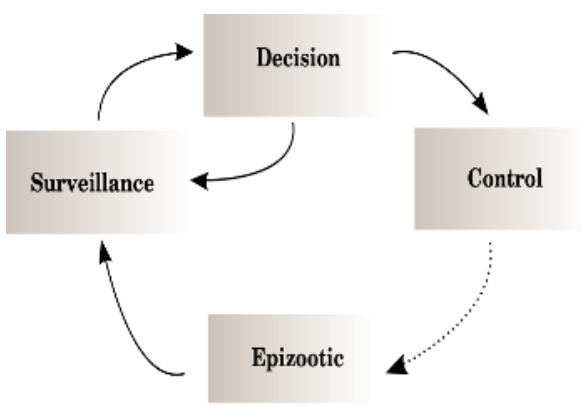

Figure 1. Schematic view of surveillance and control in animal epidemiology. The grey boxes are the main processes. The plain arrows represent the information flow. The dashed arrow symbolizes physical actions (vaccination, trading restrictions or culling). The different elements composing the surveillance, the decision, the control and the epizootic depend on the application at a particular place, for a particular time and a particular disease.

the intentional and planned surveillance preformed by official veterinarian services respectively. The interested reader can refer to the book of B. Dufour and P. Hendrikx [1] for a detailed presentation of epidemiological surveillance in animal health. In this paper, we will consider a simplified and generic version of the system by focusing on the main processes involved in surveillance and control. Figure 1 provides a schematic representation of the whole system.

The system illustrated by figure 1 exhibits the classical sensor-controller structure for the control of any physical system. One approach could be the use of the optimal control theory to study the behavior of such a system. Nevertheless, this approach is well situated for physical systems but its applicability to systems with social components is still questionable. Indeed, the decision processes in epidemiological surveillance and control, strongly depends on factors such as the social acceptability of control measures and, of course, depends on economical considerations. The decision to invest or not in surveillance and control strongly relies on the social, political and economical context. For that reason, the decision makers would benefit to forecast the cost of surveillance and control under different epidemiological scenarios. In that context, simulation can be a useful tool to help decision makers.

On the accounting side, fixing a cost to the resources and services that are consumed by a particular activity is a main concern. To deal with this issue, costing systems are designed to support decision making in business management. Among the available accounting methods, the Activity Based Costing system (ABC, [2]) uses the concept of activity to estimate direct and indirect costs in the production of goods or services. ABC belongs to the set a cost estimation methods. It establishes a relationship between costs, products and services by identifying the cost drivers in a set of interdependent activities. $\mathrm{ABC}$ was revealed as complex to implement due to the dynamical nature of processes and resources spending. Furthermore, the activities can change over time. Therefore, an improved version of $\mathrm{ABC}$ was proposed, the Time-Driven ABC (TDABC, [3]). TDABC has been largely experimented in enterprises and its effectiveness has been discussed for particular sectors, such like logistic [4].

In this paper, I initiate a connection between the basic ideas supporting the TDABC, and the weighted activity formulation recently proposed by X. Hu and B.P. Zeigler [5] in the discrete events modeling and simulation research field. I illustrate this connection in the field of surveillance and control in animal epidemiology. The objective is to design a conceptual model able to compute the global 
cost of surveillance and control considering different epizootic dynamics, and different surveillance and control strategies.

\section{Method}

The Discrete EVent System specification (DEVS) can specify many classes of dynamical systems, including hybrid discrete and continuous systems [6]. By choosing the DEVS formalism to specify the structure and the dynamic of the system under study, I assume here to be able to consider a large number of systems classes. To introduce the financial cost of any activities in a DEVS specification, I first consider the definition of the weighted activity given by X. Hu and B.P. Zeigler [5]: "in a discrete event system, the weighted activity is the sum of weighted state transitions over a time interval". Then, I make an analogy between TDABC and this definition. I propose to consider the weight of the activity to be a measure of the cost of the activity. In addition, I consider the two variables of the TDABC [3]:

- The estimation of the costs per time unit to supply resources to the activities (the total overhead expenditure of a component divided by the total employee time available).

- The amount of time necessary to carry out one unit of each kind of activity (as estimated or observed).

The second variable is computed by the DEVS time advance function. It depends on the state of the model or it can be a constant value. Similarly, the first variable of the TDABC can be a constant value changing from one simulation to another or a function depending on the state of the model. For both variables, the DEVS formulation add some potential to the expressiveness of the TDABC model.

The formalization of the weighting transition functions should be elaborate with the decision maker. We first estimate the costs per time unit, $c_{r} \in s$, for all the activities modeled as state transition function in the DEVS model. The weighting transition functions, $w_{t}$, can be specified as follow: $w_{t}: S \rightarrow R$. From here, three different cases can be identified for this function. Firstly, some transitions (activities) are performed at no cost, then $w_{t}(s)=0$. Secondly, as I wrote before, the cost can depend on the duration of the activity, in that case $w_{t}(s)=f(s, e)$, with $c_{r} \in s$ the set of state, $f()$ a weighting function and $e$ the elapsed time in the activity. Thirdly, the cost can be invariant in time, i.e. stationary, and depend only on the model state, then $w_{t}(s)=g(s)$, another weighting function. Finally, we can defined the total cost $c$ of the activities in the system at time $t+e$ as follow:

$$
c(t+e)=f(s(t), e)+g(s(t))+c(t)
$$

It is a strictly positive monotonic function. It is then straightforward to compute the cost growth rate $\alpha$ in any time interval $\left[t_{1}, t_{2}\right]$, for any components, with the following formulation:

$$
\alpha=\frac{c\left(t_{2}\right)-c\left(t_{1}\right)}{t_{2}-t_{1}}
$$

the total cost $c$ and the rate $\alpha$ can be used to compare the cost of activities in different components or DEVS models using simple ratios. It is then possible to compute the cost-efficacy ratio of a modeled systems regarding alternatives.

\section{Example}

In the following, I illustrate the proposed method in the context of an epidemiological surveillance and control system. I consider such a system as a dynamical system. Therefore, I can use DEVS as I 
state in the previous section. As for any dynamical system, we can model it with the appropriate level of details depending on the knowledge we have about the system. The objective here is to exemplify the proposed method in a didactic way that is why the system presented here is over simplified for that purpose and why I do not detail the different components. Consequently, I assume that the system presented figure 1 exhibits the necessary and sufficient level of detail for the purpose of this paper.

The system has four components: decision, control, epizootic and surveillance. The final user of the model is a decision maker. She/he should be able to choose a model for all the components. This can be done by using a component based approach for modeling and simulation. This approach is implemented in the Virtual Laboratory Environment (VLE) for instance, a set of tools to design, simulate and analyze DEVS models [7]. By choosing different models for the different components, and by setting up the required values for the parameters, the decision maker builds scenarios and can evaluate the consequences of here/his decisions on meaningful indicators (size of the epidemic, effectiveness of a particular control measure, etc.). The component "decision" models the set of rules that are a priori defined by the decision maker. Therefore, such a component does not embed any process to infer the rules to apply (the decision maker define them), rather the set of rules themselves. Figure 2 illustrates the classical cycle where decision makers are embedded when using simulations to test scenarios. It illustrates the possibility for decision makers to choose models of components from a repository. Then, regarding the simulation results, they design a new scenario to test new strategies.

Assuming that the decision maker is interested in three main indicators to make a decision, let say the prevalence of disease (the number of sick or dead animals at a particular date), the incidence (the rate at which the disease spread within the population within a defined time interval) and the cost efficiency ratio of surveillance and control. Other important indicators may exist defining the political and economical context. They are not explicitly considered. Indeed, the decision maker is part of the decision loop (figure 2), and I assume that, if she/he knows these factors, she/he will implicitly take them into account to make a decision. The first two main indicators, prevalence and incidence, are computed directly from the simulation results of the epizootic model. Before defining how to compute the cost-efficiency ratio, I describe the main processes that should be modeled in the epizootic, surveillance, decision and control components. I consider the classical definition of a process, i.e. a sequence of activities or events ordered in time.

The epizootic component:

- Receive perturbations from the control component.

- Compute the evolution in time of the disease spread using a continuous or discrete time model (for instance the classical A.G. McKendrick and W.O. Kermack epidemiological SIR model (Kermack and McKendrick [8]) or a discrete model of the disease spreading on a network, etc.).

- Send the current number of susceptible, infectious and recovered or removed animals at a frequency set by the decision maker.

The surveillance component:

- Receive the current number of susceptible, infectious and recovered animals.

- Receive the surveillance effort from the decision component (proportion of the population the surveillance system which can sampled by unit of time).

- Take a sample in the whole population regrading surveillance effort.

- Estimate the prevalence and the incidence of the disease from the sample.

- Send the prevalence and the incidence at a frequency set by the decision maker.

The decision component: 


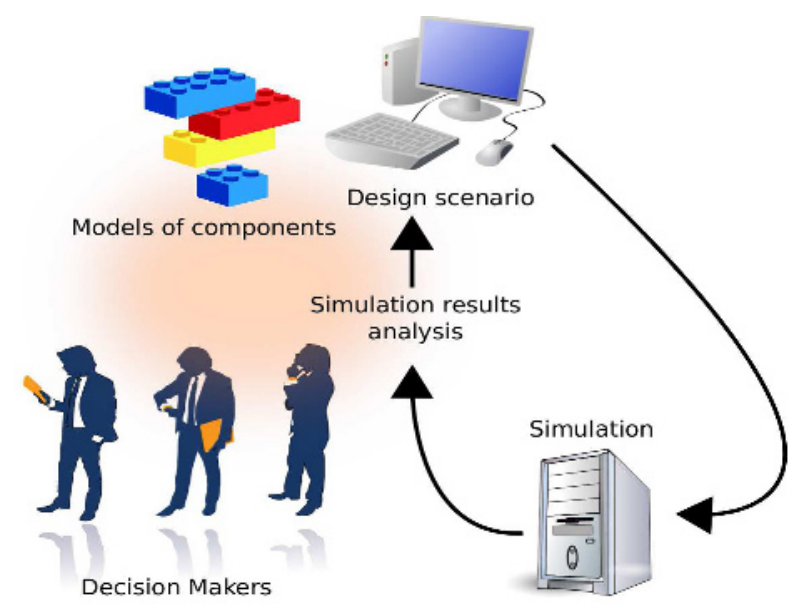

Figure 2. The iterative cycle of decision making when using simulations. Decision makers can choose the model of components regarding simulation results to design a new scenario for evaluation.

- Receive the prevalence and the incidence.

- Make a decision to reinforce surveillance and/or control regarding certain thresholds.

- If needed, inform the surveillance component of the new surveillance effort.

- If needed, activate the control component sending the chosen control strategy (vaccination, trading restrictions or culling) and the associated effort (how many individuals to vaccinate, where to perform culling or which commercial routes to cut).

The control component:

- Activated by the decision component with a particular control strategy and effort.

- Apply the control regarding the effort by sending perturbations to the epizootic component.

The financial cost is exogenous of the system. It is a constraint for the decision maker. Following the definitions given in section 2, the decision maker can follow the evolution of the cost of surveillance and control observing $c(t)$. Then, the cost efficiency ratio $E(t)$ can be estimated considering the computed cost $c^{*}(t)$ associated with the number of sick or dead animals in the abscence of surveillance and control, $c_{s c}^{*}(t)$, the cost associated with the number of sick or dead animal when applying surveillance and control and $c_{s c}(t)$, the computed cost of surveillance and control activities. $E(t)$ is written as follow:

$$
E(t)=\frac{c_{s c}(t)+c_{s c}^{*}(t)}{c^{*}(t)}
$$

This ratio can be used to compare different surveillance and control policies, and then improve decision making.

As the estimation of the costs per time units can be uncertain for some activities, the decision maker may test different surveillance and control strategies to minimize the rate $\alpha$ rather than the costs themselves. This method can then be usefull even in uncertain financial contexts. 


\section{Discussion}

Costing is a tricky issue in epidemiological surveillance and control since there is no clear evidence of the benefits brought by one strategy compared with another since we can not test alternative scenarios for practical and ethical reasons. Therefore, the example of surveillance and control in animal epidemiology illustrates a great potential of using TDABC concepts in a DEVS specification. We can simulate a situation without surveillance nor control, and estimate the economic loss for farmers or for the country, regarding the size of the epidemic for instance (number of cases). Then, using the method described above, we can compare different scenarios in term of costs of surveillance and control by quantifiying the cost efficiency compared with a situation without any intervention. Of course, The precise definition of the functions $f()$ and $g()$ is challenging. It requires a lot of expertise and the collaboration amoung specialists in the field of economy in animal health. It can be difficult to consider all the type of costs in surveillance and control. Nevertheless, considering that the main costs are correctly identified, $\alpha$ can be a good proxy of the financial effort and $c$ an indicator of its magnitude.

The decision model could be specified as "cost aware" sensor, following the example of the "energy aware" sensor described in [5]. Nevertheless, the decision process is complex here, and I think it is useful in the surveillance and control system to introduce the decision maker within the optimization loop. This inclusion presupposes the existence of many types of models usefull for the decision makers, or the existence of tools for the design and implementation of new components that could be used by the decision makers. Such tools are currently not existing, and a close interaction between the modelers and the decision makers is still needed to improve existing components and develop the new ones.

In this paper, I presupposed that some of the state transitions match activities of the real system, and that the associated costs are known. As a consequence, the DEVS abstract simulators should be modify to take into account costing. This should be done in a similar manner than for energy aware information processing [5].

\section{Conclusion}

In this paper, I initiate a discussion in concern with the use of the activity paradigm for the estimation of costs in dynamical systems with feedback loops. It is a position paper and a lot of work remains to be done to formalize the method and to test it. Nevertheless, I think there is a real potential for this method to be applied in the context of systems that can not be experimented, such as economical systems or socio-ecosystems. Such a proposition should be included in the framework we proposed with B. Bonte and J.P Müller [10]. In such a framework, the classical experimental approach is contextualized using DEVS. It enables to test the accuracy and usefulness of the models we use to make decisions on the real systems. Therefore, I think the method initiated here goes far beyond epidemiological studies. It can be apply to any systems with feedback loops and including decisions and time varing costs, even if the costs are not precisely known.

\section{References}

[1] B. Dufour and P. Hendrikx. Epidemiological Surveillance in Animal Health. FAO 2dn eds. (2009) $386 \mathrm{p}$.

[2] R. Cooper and R. S. Kaplan. Measure Costs Right: Make the Right Decisions. Harvard Business Review, 5, 96-103 (1988). 
[3] R. S. Kaplan and S. R. Anderson. Time-Driven Activity-Based Costing. Harvard Business Review, 131-139, 82, 11 (2004).

[4] P. Everaert, W. Bruggeman, G. Sarens, S. R. Anderson and Y. Levant. Cost modeling in logistics using time-driven ABC: Experiences from a wholesaler.International Journal of Physical Distribution \& Logistics Management, 3, 38, 172-191 (2008).

[5] X. Hu and B.P. Zeigler. Linking Information and Energy - Activity-Based Energy-Aware Information Processing. Simulation, 4, 89, 435-450 (2013).

[6] B.P. Zeigler, H. Praehofer, T.G. Kim, Theory of Modeling and Simulation, 2nd Edition, Academic Press (2000) 510 p.

[7] G. Quesnel, R. Duboz and É. Ramat. The Virtual Laboratory Environment - An operational framework for multi-modelling, simulation and analysis of complex dynamical systems. Simulation Modelling Practice and Theory, 17, 641-653 (2009).

[8] W. O. Kermack and A. G. McKendrick. Contributions of mathematical theory to epidemics. Proceedings of the Royal Society Series A, 115, 700-721 (1927).

[9] A. Muzy and D. Hill. What is New with the Activity World View in Modeling and Simulation? Using Activity as a Unifying Guide For Modeling and Simulation. Proceedings of the Winter Simulation Conference, IEEE/SMC-ACM/SIGSIM, S. Jain, R.R. Creasey, J. Himmelspach, K.P. White and M. Fu eds, 13 (2011).

[10] B. Bonté, R. Duboz and J.P. Müller. Modeling the Minsky Triad: A Framework to Perform Reflexive M\&S Studies. Proceedings of the Winter Simulation Conference, IEEE/SMCACM/SIGSIM, Berlin, Germany, (2012). 